\title{
ARTICOLI
}

\section{Potenzialità glottodidattiche della comunicazione pubblicitaria}

\section{Researching the Potential of Advertising Communication in Language Teaching}

\author{
Marcello Giusto \\ Università Adam Mickiewicz, Poznań \\ marcello.giusto@amu.edu.pl
}

\begin{abstract}
The aim of the present article is to shed the light on educational aspects which can be obtained by the exploration of an authentic, advertising material. The analysis attempts to demonstrate how communicative and linguistic characteristics, argumentative patterns, rhetorical figures and cultural aspects, which constitute the complex structure which is advertising message, convey values, stereotypes, social and linguistic tendencies. All of the above are conceived in order to complete the goals of persuasion. For the teacher, it is a rich, authentic and precious resource which gives the student the opportunity to extend his abilities and skills, to face and approach a new, linguistic and cultural context.
\end{abstract}

Keywords: advertising, authentic material, teaching, rhetoric

\section{INTRODUZIONE}

In questo articolo si analizzeranno non solo gli strumenti persuasivi retorici utilizzati in pubblicità come mezzi espressivi ma anche i livelli più intrinsechi nei quali essa veicola valori impliciti. Questi elementi comunicativi, linguistici, retorici e culturali presenti nel materiale autentico pubblicitario rappresentano una ricca e malleabile fonte da cui attingere potenzialità didattiche. Prima di entrare nel cuore della questione, è necessario inquadrare il concetto di materiale autentico ricordandone varietà e proprietà pedagogiche. 


\subsection{Materiale autentico: nozione e tipologie}

Il concetto di materiale autentico compare per la prima volta nelle opere di Wilkins $(1975,1976)$ e si riferisce a testi tratti da contesti reali - «real-life texts» (Wallace, 1992, p. 145) - indirizzati ad un pubblico madrelingua, dunque testi che non sono stati concepiti per stranieri e con fini didattici (Wilkins, 1976, p. 79). Morrow (1977, p. 13) precisa che un testo autentico non solo costituisce un frammento del linguaggio reale, prodotto da un parlante o uno scrittore reale per un pubblico reale, ma è anche predisposto a convogliare un certo messaggio reale che permette di simulare situazioni originali e della vita quotidiana, aumentando il livello di motivazione del discente. Per questo l'approccio comunicativo predilige questo tipo di materiale.

Begotti (2008) propone una catalogazione del materiale autentico in:

- cartaceo: testi di letteratura, articoli di giornale, pubblicità su riviste e quotidiani, cartelloni, avvisi, nonché la cosiddetta letteratura grigia. È il materiale più utilizzato dai docenti poiché facilmente reperibile, per la flessibilità d'utilizzo ed elaborazione, oltre ad essere positivamente accettato da parte dell'apprendente;

- audio: programmi radiofonici, giornali radio, pubblicità radiofonica, così come brani musicali di cantanti italiani o audiolibri;

- audiovisivo: programmi televisivi (telegiornali, quiz, talk show, previsioni del tempo, documentari, relazioni sportive, ecc.), film e pubblicità disponibili su supporto DVD;

- multimediale, sia audio che video, ovvero quello disponibile, utilizzabile e scaricabile in rete (posta elettronica, chat e social media), oppure fruibile su computer o dispositivi ad esso collegabili.

\subsection{Centralità dell'utilizzo di materiali autentici nella didattica LS/L2}

Un materiale diventa autentico nel momento in cui diviene «ciò che gli studenti e gli insegnanti potranno e vorranno farne in quel momento, esattamente come il dialogo previsto dal libro di testo o il dialogo improvvisato in aula tra studente ed insegnante» (Vassallo, 2006). Di conseguenza il docente imposterà la lezione puntando sullo sviluppo di determinate competenze e considerando ogni materiale didattico come un contenuto di variegati significati dipendenti da macro- e microcontesti all'interno dei quali vengono proposti.

È tuttavia possibile individuare aspetti negativi dell'utilizzo di materiale autentico nella didattica di una lingua straniera. Per lo studente può costituire un elemento di difficoltà legata alla comprensione linguistica e culturale, con conseguente demotivazione. D'altro canto, il docente dovrà dedicare molte risorse per l'individua- 
zione, l'analisi, la selezione e l'elaborazione del materiale autentico adatto agli scopi prefissi e alle necessità degli apprendenti. Tuttavia, a differenza del materiale didattizzato offerto dai manuali - la cui maggiore facilità di comprensione avvantaggia gli studenti - il materiale autentico rappresenta un'immagine reale ed aggiornata della cultura e del repertorio linguistico ed è dunque adeguato alla costruzione di lezioni che mirino alla comprensione e all'interazione con il mondo di quella realtà linguistica e culturale, attivando la motivazione e consentendo al docente la predisposizione di percorsi didattici ad hoc adattati alle esigenze, agli obiettivi e alle competenze degli apprendenti. Santipolo (2014, p. 16) auspica un ampliamento dell'approccio dell'insegnante verso quello che definisce bespoke language teaching, ovvero una didattica creata appunto su misura intorno all'allievo.

\section{LA PUBBLICITÀ COME MATERIALE AUTENTICO}

Il compito della comunicazione pubblicitaria è informare il pubblico attraverso i mass media (stampa, affissione, radio, cinema, televisione o i dispositivi tecnologici che operano via internet) dell'esistenza sul mercato di un determinato prodotto, delle funzioni e dei valori che lo caratterizzano e lo distinguono dalla concorrenza (Pittèri, 2001, p. 468). Per realizzare questi obiettivi ogni forma pubblicitaria si arma di strategie persuasive che mirano a suggestionare, influenzare, coinvolgere, spingere all'acquisto e fidelizzare il consumatore.

Guardando al contenuto, ogni comunicato pubblicitario veicola in primis una serie di riferimenti rispondenti a bisogni emotivi e culturali specifici, nonché lingua, gusti, caratteristiche, attitudini, valori, attualità e storia di un Paese. Si tratta infatti di materiale autentico che racconta lo spaccato di una nazione, trasmettendo inoltre importanti informazioni riguardanti l'ambito socioculturale (Pavan, 2011, p. 126). Per questi motivi il docente non dovrebbe trascurare tale materiale, ricordando che saper leggere dentro e oltre il messaggio pubblicitario può rivelarsi utile per l'apprendimento di una lingua straniera nonché per poter avvicinare lo studente alla nuova realtà, cogliendone modelli linguistici e culturali relativi a svariati ambiti. La pubblicità è quindi adatta alla didattica principalmente per tre motivi: per la familiarità dell'apprendente con questa forma comunicativa universale, con la quale ha a che fare quotidianamente; per la sua brevità e chiarezza discorsiva che permettono di essere letta/visionata più volte, facilitando la memorizzazione e la comprensione, anche grazie alla presenza di elementi non verbali che completano il messaggio audio; per la forza motivazionale dovuta alla sua spettacolarità (specie nel caso delle pubblicità televisive) e all'affinità con le altre forme di intrattenimento. 


\section{LA PUBBLICITÀ COME MATERIALE GLOTTODIDATTICO}

\subsection{Pubblicazioni a disposizione del docente}

Molti studiosi (fra cui Mollica, 1979; Desideri, 1996; Lavinio, 1997; Bonvino, 2004; Caiazzo, Liotto, Morlicchio, 2005; Spinelli, 2005; Benucci, 2007; Pavan, 2011; Ghelli, 2014) hanno più volte ricordato come l'utilizzo didattico dei comunicati pubblicitari sia positivamente recepito dagli apprendenti, motivandoli al lavoro e mettendoli a contatto con la lingua e la cultura straniera.

Chi scrive ha proceduto ad esaminare una quindicina fra le pubblicazioni di maggiore diffusione sul mercato ${ }^{1}$ e ha tracciato una mappa del materiale a disposizione del docente riguardante la pubblicità o che sfrutta testi autentici pubblicitari. È interessante innanzitutto osservare come siano rare le pubblicazioni didattiche mirate esclusivamente all'insegnamento dell'italiano tramite materiali autentici pubblicitari (si veda la serie L'italiano con la pubblicità, pubblicata dall'editore Guerra fra il 2004 e il 2005). Più sovente, infatti, i manuali utilizzano come input (brevi) annunci e manifesti pubblicitari (spesso autentici), nonché prodotti (tipici) italiani, già nei volumi destinati ai principianti. All'innalzamento del livello corrisponde ovviamente un grado maggiore di difficoltà, in linea con lo sviluppo delle competenze linguistiche. Si trovano infatti attività di comprensione globale e dettagliata di annunci radiofonici, di offerte pubblicitarie online e di spot. Ad ogni livello, ogni materiale autentico è spesso sfruttato per introdurre temi grammaticali di varia natura oppure come modello comunicativo da imitare. Nei testi per studenti avanzati le attività correlate mirano ad approfondire aspetti linguistici e sociali, toccando tematiche attuali e richiedendo un maggiore pensiero critico. In due manuali la pubblicità è il tema centrale del percorso didattico: Viaggio nell'italiano - Cl (Unità 7) e Nuovo Contatto Cl (Unità 1 - Percorso 4). Proprio in quest'ultimo troviamo materiali di diversa natura (spot in formato video, manifesti a stampa, audio) i quali, dopo la visione, lettura e comprensione, vengono utilizzati come stimolo per esprimere opinioni sui messaggi che intendono trasmettere, per un'analisi delle strategie sociali, per riflettere sugli slogan, sul valore e ruolo della famiglia, conoscere meglio la Pubblicità Progresso, analizzare il linguaggio pubblicitario. Inoltre, sono indirizzati all'approfondimento di determinati elementi grammaticali, lessicali, sociolinguistici (introduzione ai dialetti) nonché culturali (esercizio di completamento sulla storia di Carosello). Questo manuale può essere considerato un'interessante fonte dalla quale attingere materiale pronto e ispirazione per l'elaborazione di nuove risorse didattiche.

\footnotetext{
${ }^{1}$ Affresco Italiano, Ascolto Avanzato, Bravissimo! 3, In Itali@ 2, Io \& 1'italiano, Italia per stranieri, La formazione del lettore (forme e tipi di testi), La lingua e i testi - Vol. B, Linea Diretta 1, Nuovo Contatto C1, Nuovo Espresso 6, Nuovo Progetto Italiano 1 e 2, Primo Contatto, Viaggio nell'Italiano.
} 


\subsection{Potenzialità e aspetti didattici dei materiali pubblicitari autentici}

Per costruire un messaggio persuasivo i pubblicitari sfruttano ogni livello espressivo-comunicativo a seconda del medium prescelto con riflessi sui contenuti. Questi possono essere analizzati e compresi attraverso uno studio che guidi l'apprendente ad una lettura degli elementi costitutivi. Sfruttando dunque pubblicità autentiche, il docente ha la possibilità di colmare o completare la lacuna dei materiali disponibili sui libri di testo decidendo, conformemente al livello linguistico, su quale aspetto o su quali elementi rivolgere l'attenzione degli studenti. Trattandosi di materiale autentico, sarà necessario predisporre delle attività preliminari che agevolino la comprensione, dapprima globale e poi gradualmente selettiva ed analitica (Harrich, 2011, p. 334). Si avvicinerà così il discente al tema del messaggio e a quegli elementi comunicativi, linguistici e culturali a lui/lei sconosciuti, che sono invece naturali e scontati per il pubblico madrelingua.

Prima di predisporre un piano canonico della lezione secondo la scansione motivazione, globalità, analisi, sintesi, riflessione, rinforzo e approfondimento (cfr. Balboni, 2002), il docente deve compiere una ricerca del materiale pubblicitario autentico a disposizione che più si adatti ai propri scopi didattici, passando, si è detto, attraverso le fasi di individuazione, analisi, selezione per poi giungere alla sua elaborazione. Fra le forme pubblicitarie più appropriate e ampliamente disponibili ci sono gli spot (facilmente rintracciabili su YouTube o cercando nei contenuti multimediali presenti nei siti internet di alcuni produttori) e i manifesti o gli annunci a stampa (reperibili pressoché su ogni quotidiano o rivista in commercio). Per una migliore selezione del materiale, oltre alla rispondenza con gli obiettivi didattici prefissati, il docente dovrebbe: selezionare con attenzione il materiale per i livelli principiante ed elementare, controllare la presenza di varietà linguistiche, valutare la difficoltà del lessico, notare la possibilità di creare attività didattiche in forma di unità, cogliere le relazioni fra forma tecnica e contenuto del messaggio, fare attenzione alla qualità del sonoro, prevedere altri problemi che potrebbero impedire la comprensione, testare e rielaborare nel tempo il materiale didattizzato (Begotti, 2010).

Per trasformare le pubblicità in materiale didattico il docente considererà ogni aspetto comunicativo, linguistico, retorico-argomentativo nonché culturale racchiuso all'interno dei diversi livelli che compongono il messaggio.

\subsubsection{Aspetti comunicativi}

Per comprendere meglio le dinamiche della pubblicità considerata come tipologia di comunicazione, è essenziale stabilire quali siano le sue componenti fondamentali. Nel nostro caso, emittente e destinatario sono rispettivamente pubbli- 
citari/produttori e consumatori. Lo spazio in cui avviene l'atto comunicativo può essere distinto in setting e scene (Hymes, 1974, p. 55), ovvero il contesto reale che il consumatore vede/sente rappresentato nella pubblicità e quello di fantasia che il comunicato promozionale intende in lui/lei rievocare facendo leva su determinati elementi psicologici e culturali. A questi Pavan (2011, pp. 135-137) aggiunge: il luogo nello spazio (il setting visibile nel quale avviene l'atto comunicativo e in cui il parlante ha un ruolo sociale o privato), il luogo nel tempo (il momento o l'occasione che fa da contesto), il luogo nella mente dei partecipanti (la loro situazione psicologica, ovvero il loro stato d'animo e il ruolo ricoperto nella cornice comunicativa), i partecipanti (ogni persona coinvolta nella comunicazione, ciò che in essa rappresenta, la sua funzione e le aspettative degli altri), la comunicazione, verbale $e$ non verbale (oggetti, testi, scritti e orali, nonché ogni elemento prossemico, cinesico, tattile ecc. dei partecipanti, spesso diversi a seconda della cultura).

Osservando la comunicazione pubblicitaria attraverso lo specchio delle funzioni comunicative di Jakobson, essa si avvale, anche nel contempo, di ognuna di esse. Più sovente ricorrono quella referenziale-informativa (Il dentifricio Colgate contiene gardol), quella espressiva (Io sono Giulietta e sono fatta della stessa materia di cui sono fatti $i$ sogni) e conativa (Passa a Vodafone!), orientate al destinatario.

\subsubsection{Aspetti linguistici}

Il linguaggio pubblicitario è definito da diversi studiosi come una lingua speciale in senso lato, ovvero una varietà linguistica ai margini (cfr. Migliorini, 1963; Berruto, 1987, p. 154 e segg.; Sobrero, 1993, p. 237 e segg.; Dardano, 2003, p. 199 e segg.), come una lingua artificiale, autonoma rispetto alla lingua madre poiché possiede proprie regole create da un illimitato estro (Cardona, 1974, p. 34), caratterizzata da un uso non naturale, che sfrutta tratti del parlato informale o riferimenti intertestuali (Rossi, 2011, pp. 1187-1188). È evidente come lo si possa considerare «una sorta di laboratorio linguistico» (Capozzi, 2008, p. 85) nel quale l'apprendente potrebbe ricercare ad un livello più dettagliato le ricche risorse espressive che si esaminano qui di seguito.

In principio, si farà notare agli studenti la manipolazione di grafie, punteggiature e caratteri utilizzata spesso per marchi o logotipi ad es. tramite amplificazioni e riverberi di suoni che attivano associazioni mentali universali (cfr. Dogana, 1991, p. 38), come nel caso di nomi di biscotti e cracker che rievocano la croccantezza (Frosties, Sbricioline). Una strategia non solo italiana è inoltre quella di elevare il nome di un prodotto e conferirgli una certa scientificità usando le (pseudo) lingue classiche: detersivo Aiax, elettrodomestici Ariston, prodotti per la pelle Nivea e Venus, gelati Magnum, surgelati Findus, biscotti Plasmon e le auto Alfa, Delta, 
Gamma, Omega (anche marca di orologi), Ypsilon ecc. (Rossi, 2011, p. 1187; Arcangeli, 2008, p. 73).

Nel lessico delle pubblicità ricorrono inoltre forestierismi che riflettono «fedelmente lo status e il prestigio che si associano [...] a una determinata lingua straniera» (Cardona, 1974, p. 61), comprese le associazioni stereotipiche ad esse collegate. In particolare, il francese è la lingua della moda, dell'eleganza, della raffinatezza e della bellezza, adatta a prodotti cosmetici e di abbigliamento (YvesSaint Laurent: l'homme sensuel et magnétique); l'inglese è sinonimo di modernità ed efficienza, di un alto status sociale e il suo prestigio riflette l'identità cosmopolita del consumatore del mercato globale (Pandora / Unforgettable moments) arrivando inoltre a garantire l'italianità dei prodotti (Nobili / Cashmere Made in Italy); lo spagnolo, benché meno frequente, può essere usato per evocare uno stile giovane e dilettevole (Seat. Auto emoción). Talvolta ricorrono anche altre lingue o mescolanze di vari forestierismi all'interno dello stesso enunciato, con effetti esagerati e divertenti: Molto Summer, poco Lease, very Kool. / Nuova SLK Chrome e Sport; Pupa / Non conventional Beauty. / Ombretto à la coque. / Ombretto cotto / Doppio utilizzo (cfr. Capozzi, 2008, pp. 102-107).

Al fine di rendere più autorevole, credibile il messaggio e di infondere fiducia nel compratore, vengono impiegati tecnicismi, come clinicamente testato, lactobacillus bifidus essensis, o tratti dal gergo militare-guerresco in senso metaforico: Lifactiv supreme per combattere giorno dopo giorno la comparsa dei segni del tempo (Capozzi, 2008, pp. 87-88).

In campo morfologico si nota un ampio utilizzo dei superlativi assoluti (Meglio Bosch, perché Bosch è migliore) a cui fa eccezione - con conseguente effetto straniante dovuto alla violazione della consuetudine - lo slogan il rum più bevuto nei peggiori bar di Caracas. Si troverà sovente il modo imperativo, usato per persuadere più direttamente il destinatario, riferendosi ad emozioni o azioni legate a un beneficio, come nello slogan di Mulino Bianco Barilla: Mangia sano e vivi bene.

Formazione di parole e neologismi sono impiegati per sintetizzare concetti e rimarcare $\mathrm{o}$ amplificare precise qualità del prodotto. Ricorrono processi di:

- composizione tramite i prefissi elativi super-, iper-, extra-, ultra-, nonché anti-, mini-, maxi-, pluri-, bio-, eco- creando espressioni come supermoderno, ultrasensibile, mini-prezzo, maxicono, antimacchia, antirughe, anti-età, biotecnologico, ecopelle ecc.; uso esasperato del suffisso -issimo tipico del superlativo assoluto: bellissimo, elevatissima, morbidissima, pregiatissimo, ecc. fino al famoso slogan dell'acqua minerale Altissima, purissima, Levissima;

- apposizione del suffisso -oso 'avente caratteristiche di $\mathrm{X}$ ' con risultati stravaganti (comodoso, risparmioso, viaggioso); uso dei suffissi -evole, -icante e -icida (amarevole 'liquore amaro dal gusto gradevole', amaricante 'dal gusto non troppo deciso', odoricida 'un deodorante dal profumo delicato ma potente come un insetticida'); 
- deverbalizzazione creativa (insalatare, vespizzatevi, cioccolatarsi, benzinare);

- convergenza di varie parole con una base verbale (detersivo brillatutto, pannolini aiutamamma, ombrelli sfidapioggia);

- giustapposizione di due o più elementi (modello-famiglia 'modello per la famiglia', lanagiovani 'lana per giovani', boccasana, doppiobrodo, piuchepanettoni ecc.);

- parole-macedonia, composte eliminando parte degli elementi costituenti e il cui significato finale è dato dalla somma logica delle due parole originarie (ultimoda $[$ ulti $(m a)+$ moda $]$, erbamaro $[$ erb $(a)+$ amaro] 'amaro a base di erbe') fino agli estremi semplologica [semplice + tecnologica], modtro [moderna + retrò] e brillosa [brillante + vantaggiosa] della serie di pubblicità automobilistiche per la nuova Micra.

A livello sintattico, si nota l'uso dell'articolo con funzione enfatica e valore di unicità (Illy, il caffè perfettamente espresso; un film Paramount) o la sua omissione (Amaro Montenegro, sapore vero). Inoltre si riscontrano:

- costruzione comparativa con cancellazione del secondo termine di paragone (Fresco, più fresco, tre volte più a lungo);

- stile nominale, forme ellittiche e giustapposizione asindetica per creare soluzioni sintetiche ed efficaci (Tante vite, un solo appuntamento. Fiat) rese anche con omissione di preposizione (Ava lavatrice; tè filtro);

- rematizzazione con spostamento dell'elemento novità in prima posizione in modo da evidenziarlo (C'è un nuovo pulito su tutto il bucato: Ace Lavatrice) e con frasi scisse che riproducono il parlato ( $\dot{E}$ così che piaccio alle donne: rapace. Emporio Armani occhiali);

- tematizzazione tramite sospensione del tema (Cipro. Che spiaggia di Cipro state sognando?), dislocazione a sinistra (Saeco Magic. Il caffè lo prendiamo a casa) o a destra (Lo vuoi vedere il cuore?);

- instaurazione di un'illusoria interazione diretta con il consumatore mediante periodi ipotetici che sembrano lasciargli un'apparente libertà di scelta ( $\mathrm{Se}$ osassi lasciarmi andare, che male ci sarebbe? J'adore), frasi interrogative (Quattro giga, o pranzo con me?), enunciati imperativali (Bevi Coca-Cola).

In una prospettiva sociolinguistico-culturale, negli ultimi anni, grazie alla tradizione cinematografica e la diffusione della televisione, la pubblicità ha sfruttato in maniera crescente elementi diastratici spesso più informali e colloquiali, dando sempre più spazio a tratti (semi)dialettali o accenti regionali. In questo modo il messaggio si fa più empatico e vicino alla realtà del consumatore, stabilendo una diretta relazione fra prodotti e origine geografica: Nella pizza, 'o Sole e niente cchiù (della ditta 'O sole 'e Napule), Se Milano avess lu mer, sarebb 'na piccola Ber (criptocitazione del motto barese che Lufthansa recupera per promuovere la linea Milano-Bari). 
La pubblicità può mirare ad un'identificazione con il destinatario adattando il linguaggio a seconda dell'età e del sesso. Per le bambine si prediligono filastrocche e canzoncine ricche di vezzeggiativi (-ino, -etto), iperboli (grande, massimo, fantastico e i composti già menzionati) lessico povero e ristretto al baby talk (Ora, mentre tu fai la nanna, / la mamma ti prepara la pappa! [...] Non ti succhiare il ditino, è pronta la pappa), mentre per i maschietti le formule sono più forti e dinamiche, marcate da un ritmo serrato e dalle occlusive velari /k/ e /g/ (Scopri il gran vigore dei guerrieri Bakugan / Con chi hai a che fare? Acchiappali e combattili con le tue carte Pokemon). Si nota inoltre un largo uso di anglicismi, anche nel naming (Dragon Ball, Spiderman, Cicciobello My Phone), nonché il ricorso alle forme diafasiche e diastratiche dell'italiano giovanile e a neologismi derivati dalle nuove tecnologie negli annunci per gli adolescenti: Che aspetti? Manchi solo tu! / Ti aspettiamo sulla pagina Facebook di Top girl / clicca qui! (cfr. Capozzi, 2008, pp. 89-97).

\subsubsection{Aspetti retorico-argomentativi}

Un'analisi retorica che vada al di là della semplice identificazione di figure e tropi (Załęska, 2014) permette di indurre lo studente a riflettere su quali siano le intenzioni di un enunciato e sul modo in cui si cerca di convincere l'ascoltatore. Oltre alle scelte menzionate (imperativo come forma esortativa perentoria, uso intimistico della seconda persona, ellissi), che sono strategie retoriche sfruttate dai pubblicitari per avvicinare e coinvolgere il consumatore, esistono altri strumenti persuasivi che operano a livello inconscio.

La ricerca della concezione attorno alla quale costruire l'argomentazione (inventio) oltre a diversi loci o tópoi (luoghi comuni condivisi da tutti che permettono di sviluppare il discorso in una certa direzione) ha due modelli di riferimento. Il primo è incentrato sull'èthos, ovvero sul carattere dell'Io-emittente (la pubblicità), tipico dei messaggi informativo-referenziali. Si focalizza infatti sul marchio (Carte d'Or. Alle origini del gusto) o sulle qualità del prodotto (L'acqua Lete è pura e favorisce la diuresi). Come nel primo esempio, possono essere espresse sinteticamente in modo implicito e per mezzo di débrayage, cioè l'elisione di parti ritenute futili o ovvie, lasciando spazio all'inventiva del destinatario per completarle (Gli italiani preferiscono le bionde, metonimia anfibologica che riconduce al prodotto, la birra), oppure in maniera esplicita, in forma di ampi testi didascalici. Il secondo modello si basa invece sul pathos, sulle emozioni veementi che mirano a coinvolgere direttamente il pubblico, che ne subisce passivamente il fascino. È tipico delle pubblicità emotive costruite sull'esempio-modello da perseguire. L'esortazione diretta viene creata tramite l'uso dialettico di domandarisposta (Cerchi un addetto alle pulizie affidabile e assicurato? Rilassati con 
Helping!), imperativo alla seconda persona singolare o plurale, e aggettivi possessivi (Vai su www.helping.it e prenota la tua prima pulizia da soli 10,90 Euro l'ora).

La parte dell'argomentazione che intende delectare il pubblico (elocutio) ricorre nella compositio a svariati espedienti dell'ornatus, le figure retoriche, ovvero strumenti che arricchiscono il linguaggio e, nel contempo, suggestionano l'ascoltatore stimolando la memorizzazione, per es. grazie alla gradevolezza del testo o del suono.

Fra le figure morfologiche, basate su ritmo e suono della parola, in pubblicità si ritrovano:

- Rima: Rowenta. Per chi non s'accontenta; Crodino / l'analcolico biondo / che fa impazzire il mondo;

- Assonanza (similarità delle consonanti finali): Amadori, per veri amatori;

- Allitterazione (corrispondenza del suono consonantico iniziale in due o più parole): Chi beve birra campa cent'anni;

- Paronomasia (accostamento di parole simili ma dal diverso significato): Se c'’̀ il limone, Limoncè.

Fra le figure sintattiche, basate sulla variazione della struttura della frase con conseguenti alterazioni nell'interpretazione del significato, in pubblicità troviamo:

- Anafora (ripetizione di una o più parole all'inizio di due o più versi/enunciati): Più lo mandi giù, più ti tira su (definibile anche isocolon vista la completa equivalenza della struttura sintattica e metrica);

- Anadiplosi (dove lo schema di ripetizione è ...X/X...): Nuovo ritmo. Ritmo FIAT;

- Epanadiplosi (con schema X... / ...X): Y10. Piace alla gente che piace;

- Chiasmo (struttura a forma di croce nel quale le parole corrispondono fra loro secondo lo schema ABBA): A sporchi estremi, estremi rimedi, dove è evidente, oltre alla correlazione centrale, quella esterna con significato "rimedi allo sporco";

- Polittoto (variazione morfosintattica di elementi ripetuti): Rabarbaro Zucca Aperitivo / Che ne bevve ne ribeve. / Chi ne beve ne berrà.

I tropi di pensiero o semantici, che alterano il significato, sono anche definiti figure stranianti poiché mirano sull'effetto inatteso. In pubblicità ricorrono:

- Antonomasia (l'estensione di un nome proprio, o una perifrasi, alla definizione elementi simili, o viceversa): Non si dice Sambuca, si dice Molinari;

- Metafora (trasferimento di significato sostituendo un significante con un altro simile, è una similitudine senza spiegazione del nesso fra termini): Müller. Fate l'amore con il sapore ("lo yogurt Müller ha un sapore tale che mangiarlo è come fare l'amore");

- Sineddoche (trasferire, all'interno dello stesso campo semantico, un significato di un lemma a un altro da cui dipende reciprocamente secondo rapporti quantitativi): Pronta per un'estate perfetta? Anche i tuoi capelli lo sono! (una parte per il tutto); 
- Metonimia (sostituzione di un termine vicino con una relazione spaziale, temporale o causale): Milano da bere ("Milano" è sia "la città" sia "il luogo di produzione");

- Sinestesia (una metafora con un trasferimento di significato fra gli ambiti sensoriali dei lemmi): gusto pulito; un sogno buonissimo;

- Prosopopea (o personificazione): Enel. L'energia che ti ascolta; La scarpa che respira;

- Antitesi (esprimere un concetto evidenziando il suo contrario): Buono da diventar cattivi; Più lo mandi giù e più ti tira su (con allitterazione e anafora);

- Ossimoro (unione paradossale di termini di senso opposto): Hermés, artigiano contemporaneo dal 1837;

- Eufemismo (attenuazione di un concetto esprimendolo con una sostituzione o parafrasi): ad es. in quei giorni per indicare il ciclo mestruale;

- Litote (dire una cosa affermando l'opposto, anche con valore ironico): PIC indolor. La siringa niente male; in pubblicità è anche realizzata tramite la discordanza fra testo e immagine;

- Iperbole (esagerazione, per difetto o per eccesso, di un concetto): Tempur / $i$ materassi $n .1$ al mondo.

In pubblicità si riscontrano anche: domande (e risposte) retoriche (Nespresso. What else?), l'ironia e la criptocitazione, realizzata riadattando titoli, nomi, proverbi, frasi fatte, espressioni bibliche ecc.: L’importanza di chiamarsi Molinari; Il primo TOUCH non si scorda mai; Non avrai altro jeans al di fuori di me.

\subsubsection{Aspetti culturali}

Lo studio degli aspetti culturali di un messaggio permette di comprendere e spiegare determinate scelte creative. È proprio intorno alla pubblicità e al mondo in essa rappresentato che è possibile rintracciare i riferimenti alla storia del Paese e della televisione, a costumi, a stereotipi, a persone e alle loro preferenze, considerando anche quel bagaglio di valori che nella comunicazione è dato per scontato poiché automaticamente condiviso dai parlanti. Si pensi al binomio cappuccinocornetto che, in Italia, è un chiaro riferimento alla colazione tipica. Sostituirli con uova al tegamino e pancetta sortirebbe ben altre associazioni, a patto di non essere inglesi (Pavan, 2011, pp. 138-139). La pubblicità sfrutta infatti anche queste connessioni culturali per meglio inserire il prodotto in una determinata società, anche rinnovandosi di continuo in modo da essere al passo con il progresso tecnologico e usufruire di riferimenti attuali (ad es. gli spot degli operatori telefonici).

Volendo approfondire la storia del Paese vale la pena ricordare che gli spot moderni risentono tutt'oggi dell'eredità di Carosello, la prima forma di pubblicità televisiva italiana andata in onda per vent'anni dal 1957. Carosello ha dato vita 
a molti modi di dire che sono entrati nell'uso comune (Contro il logorio della vita moderna...; Basta la parola!; Sembra facile!) a cui si associano noti personaggi animati e del mondo dello spettacolo. Oltre ad essere illustrazione storica che ha veicolato in Italia la società dei consumi durante il boom economico, può rivelarsi un'interessante tipologia narrativa da esplorare e utilizzare in attività di storytelling o come fonte da cui trarre attività incentrate su particolari usi linguistici. Per es. nel carosello della Bialetti Telequiz della serie con l'Omino coi baffi ${ }^{2}$ si trovano espressioni comuni anche legate al mondo marino (venire incontro, avere fra le mani, andare a fondo, lupo di mare).

I temi di cui si serve la comunicazione pubblicitaria permettono di riflettere sulla visione del mondo che possiede la comunità della lingua studiata, ma offrono anche la possibilità di comparare la propria società con quella straniera sviluppando la consapevolezza degli aspetti che le differenziano. Confrontiamo due spot di IKEA in Italia e in Polonia. Il primo, Basta poco per cambiare $^{3}$, del 2012, nella sua semplicità illustra diverse modalità di abitare e vivere felicemente con qualcuno: ci sono gruppi di amici, coppie eterosessuali con bambini, una nonna single che fa yoga e, simbolo di una certa "modernità" inclusa nel titolo, una coppia di giovani omosessuali che, dopo aver unito due letti singoli, si abbraccia. L'anno successivo in Polonia il tema conduttore degli spot IKEA è Dom to frajda (La casa è uno spasso $)^{4}$, nei quali si mostra dal punto di vista dei bambini quanto arredare (e vivere) una casa possa essere divertente come un gioco. Probabilmente l'omosessualità era ancora un tema tabù nella cultura polacca, mentre in Italia iniziava a farsi più popolare (già due anni dopo verrà trasmessa la serie di spot Gustose sorprese della Findus ${ }^{5}$, dove si gioca sull'effetto sorpresa del botta e risposta finale dovuto al coming-out del figlio che al «Ci farai un figurone con il tuo coinquilino» della madre replica «Mamma, è il mio compagno»).

Elaborare una lezione che indaghi gli aspetti socio-culturali risulterà quindi fondamentale per vedere se e come la pubblicità inscena una realtà sociale ideale, con la perfetta famiglia tradizionale e felice, i nonni saggi, i figli ubbidienti, o se si tratti invece solo di un'illusione.

\section{CONCLUSIONI}

La comunicazione pubblicitaria rappresenta un ricco materiale autentico a disposizione del docente. Il mondo verosimile in essa riprodotto racconta uno spaccato di una società, utilizzando mezzi espressivi finalizzati alla persuasione. Rivolgere

\footnotetext{
${ }^{2} \mathrm{http}: / /$ bit.ly/Bialetti-telequiz. (20.05.2020).

${ }^{3} \mathrm{http}: / /$ bit.ly/IKEA-basta-poco-per-cambiare. (20.05.2020).

${ }^{4} \mathrm{http}: / /$ bit.ly/IKEA-dom-to-frajda. (20.05.2020).

${ }^{5} \mathrm{http} / / /$ bit.ly/Findus-gustose-sorprese. (20.05.2020).
} 
l'attenzione dello studente alla ricerca degli aspetti linguistici, retorici e culturali che la compongono significa comprendere i meccanismi associativi su cui si basano le scelte dei pubblicitari. Guidarlo nella scoperta della cultura, della storia e del costume di un Paese potrà essere inoltre uno stimolo per la creazione di discussioni su determinate tematiche.

Il materiale autentico deve essere scelto accuratamente dal docente, in modo da non infondere nell'apprendente un senso di frustrazione dovuto alle difficoltà di comprensione linguistiche e culturali. Elaborando un materiale autentico ad hoc, il docente potrà focalizzare l'attenzione dello studente sugli obiettivi prefissati, mediare l'attività, guidare la comprensione predisponendo appropriate attività preparatorie. Il docente selezionerà $i$ fenomeni che più ritiene adeguati ai discenti e ai propri fini didattici tenendo presente che se per il livello elementare e intermedio saranno più consone attività incentrate su particolarità grammaticali, inventiva lessicale e situazioni d'uso comunicativo (senza tralasciare il contesto storicoculturale), con gli studenti avanzati sarà possibile predisporre un'analisi retorica e lessicale delle soluzioni più complesse, individuandone processi di scelta e formazione che potranno essere messi in pratica in attività di produzione creativa.

Il vasto repertorio pubblicitario a disposizione contiene dunque molteplici potenzialità e rappresenta una fonte didattica da cui il docente può trarre quei fenomeni culturali, linguistici e sociolinguistici sui quali intende soffermare l'attenzione degli studenti, adoperandone i contenuti come spunto di discussione e di analisi critica e linguistica. Per l'apprendente si tratta di un materiale autentico i cui elementi linguistici, retorici e culturali permettono di ampliare il proprio quadro conoscitivo della realtà sociale e filologica italiana passata o contemporanea, portandolo a conoscere altresì strumenti testuali e argomentativi di riferimento utili alla realizzazione di scopi comunicativi e di produzione personale. 


\section{BIBLIOGRAFIA}

Arcangeli, M. (2008). Il linguaggio pubblicitario. Roma: Carocci.

Balboni, P.E. (2002). Le sfide di Babele. Torino: UTET.

Begotti, P. (2008). Dalla pubblicità alle canzoni: didattizzare materiale autentico per insegnare l'italiano a stranieri. Rivista ITALS, XVI, 33-50. Retrieved from: http://www.provincia.bz.it/ cultura/download/Articolo_2_Begotti.pdf.

Begotti, P. (2010). Didattizzazione di materiali autentici e analisi dei manuali di italiano per stranieri. Laboratorio ITALS. Università Ca' Foscari di Venezia. Retrieved from: http://www. itals.it/sites/default/files/Filim_didattizzazione_analisi_teoria.pdf.

Benucci, A. (2007). Lingue straniere e pubblicità: pubblicità televisiva e insegnamento dell'italia no. In M. Cardona (ed.), Vedere per capire e parlare (pp. 107-136). Torino: UTET Università.

Berruto, G. (1987). Sociologia dell'italiano contemporaneo. Roma: Carocci.

Bonvino, E. (2004). I materiali didattici per l'insegnamento dell'italiano L2: criteri di selezione. Pisa: ICON.

Caiazzo, L., Liotto, M.R., Morlicchio, E. (2005). La pubblicità anima la didattica. In C. Lavinio (ed.), Educazione linguistica e educazione letteraria. Intersezioni e interazioni (pp. 106-115). Milano: FrancoAngeli.

Capozzi, M.R. (2008). La comunicazione pubblicitaria: aspetti linguistici, sociali e culturali. Milano: FrancoAngeli.

Cardona, G.R. (1974). La lingua della pubblicità. Ravenna: Longo.

Dardano, M. (2003). Il linguaggio dei giornali: il registro pubblicitario. In M. Baldini (ed.), Le fantaparole. Il linguaggio della pubblicità (pp. 199-206). Roma: Armando Editore.

Desideri, P. (ed.) (1996). La pubblicità tra lingua e icona. Ancona: Humana Editrice.

Dogana, F. (1991). Iconismi verbali nel messaggio della pubblicità. In L. Satta (ed.), Il linguaggio della pubblicità (p. 23-43). Milano: Mursia.

Ghelli, S. (2014). Lo spot pubblicitario nell'apprendimento della "Cultura Italia". Voices in Italian Americana, vol. 25, I, 3-17.

Harrich, K. (2011). Il notiziario ovvero l'importanza dell'uso di materiali autentici nell'insegnamento-apprendimento linguistico. Italiano LinguaDue, II, 321-335.

Hymes, D. (1974). Foundations in Sociolinguistics: An Ethnographic Approach. Philadelphia: University of Pennsylvania Press.

Lavinio, C. (1997). La pubblicità nella didattica. In G.L. Quartu (ed.), Crescere con la pubblicità. Didattica e comunicazione pubblicitaria. Cagliari: Artigianarte.

Magagnoli, S. (2013). Carosello, ovvero l'educazione al consumo. Parma economica, 3, 32-38.

Mauroni, E. (2001). Imparare l'italiano L2 con le canzoni. Un contributo didattico. Italiano LinguaDue, I, 397-438.

Migliorini, B. (1963). Lingua Contemporanea. Firenze: Sansoni.

Mollica, A. (1979). A Tiger in Your Tank: Advertisements in the Language Classroom. The Canadian Modern Language Review / La Revue Canadienne des Langues Vivantes, 35, 4, 691-473.

Morrow, K. (1977). Authentic texts and ESP. In S. Holden (ed.), English for specific purposes. London: Modern English Publications.

Pavan, E. (2011). Saper osservare la pubblicità come documento interculturale. Revista De Italianística, 21-22, 123-148.

Pittèri, D. (2003). Pubblicità. In A. Abruzzese (ed.), Lessico della comunicazione (pp. 468-474). Roma: Meltemi.

Rossi, F. (2011). Pubblicità e lingua. In R. Simone (ed.), Enciclopedia dell'italiano, vol. II (pp. 1187-1188). Roma: Istituto della Enciclopedia Italiana. 
Salerno, S. (2014). Il linguaggio pubblicitario fra persuasione e retorica. H-ermes. Journal of Communication, II, 59-80.

Santipolo, M. (2014). L’impiego dei materiali autentici per lo sviluppo della competenza sociolinguistica: riflessioni teoriche e spunti operativi. Cultura e comunicazione, V, 5, 15-22.

Sobrero, A.A. (1993). Lingue speciali. In A.A. Sobrero (ed.), Introduzione all'italiano contemporaneo, vol. II (pp. 237-277). Bari: Laterza.

Spinelli, B. (2005). L'utilizzo di materiali autentici nell'insegnamento dell'italiano come LS. In $\mathrm{La}$ formazione di base del docente di italiano (pp. 133-147). Roma: Bonacci.

Vassallo, M.L. (2006). L'ippogrifo didattico: il fascino del materiale autentico. Bollettino Itals, 14. Retrieved from: https://www.itals.it/l'ippogrifo-didattico-il-fascino-del-materiale-autentico.

Wallace, C. (1992). Reading. Oxford: Oxford University Press.

Wilkins, D.A. (1975). A communicative Approach to Syllabus Construction in Adult Language Learning. In A.J. van Essen, J.P. Menting (eds.), The context of Foreign Language Learning (pp. 173-179). Assen: Van Gorcum.

Wilkins, D.A. (1976). Notional syllabuses. Oxford: Oxford University Press.

Załęska, M. (2014). Retoryka i glottodydaktyka. Perswazja na zajęciach z języka włoskiego. Język Obcy w Szkole, 3, 35-39. Retrieved from: http://jows.pl/sites/default/files/zaleska.pdf.

\section{Materiali audiovisivi}

Carosello Bialetti, serie Sembra facile, episodio “Telequiz" (1958), http://bit.ly/Bialetti-telequiz. Spot Findus, serie Gustose sorprese (2014), http://bit.ly/Findus-gustose-sorprese.

Spot IKEA Italia, serie Basta poco per cambiare (2012), http://bit.ly/IKEA-basta-poco-per-cambiare. Spot IKEA Polska, serie Dom to frajda (2013), http://bit.ly/IKEA-dom-to-frajda. 